\title{
Review
}

\section{Best Practices and Standard Protocols as a Tool to Enhance Translation for Neuromuscular Disorders}

\author{
Raffaella Willmann ${ }^{\mathrm{a}, *}$, Annamaria De Luca ${ }^{\mathrm{b}}$, Kanneboyina Nagaraju ${ }^{\mathrm{c}}$ and Markus A. Rüegg ${ }^{\mathrm{d}}$ \\ ${ }^{a}$ Swiss Foundation for Research on Muscle Diseases, Cortaillod, Switzerland \\ ${ }^{\mathrm{b}}$ Unit of Pharmacology, Department of Pharmacy and Drug Sciences, University of Bari “A. Moro,” Bari, Italy \\ ${ }^{\mathrm{c}}$ Center for Genetic Medicine Research, Children's National Medical Center, Washington, D.C., \\ United States of America \\ ${ }^{\mathrm{d}}$ Biozentrum, University of Basel, Basel, Switzerland
}

\begin{abstract}
Recent years witnessed an exciting increase in the number of clinical trials for neuromuscular disorders, in particular for Duchenne Muscular Dystrophy and Spinal Muscle Atrophy. Given the high emotional impact of such developments for devastating diseases with an urgent medical need, it is particularly important to justify human trials on the basis of robust preclinical studies and to avoid a waste of hopes and of funds.

This review focuses the discussion on the quality in the conduct clinically-oriented preclinical assessments in rare neuromuscular disease models and on the importance in reporting of preclinical confirmatory studies. Accordingly, it invites scientists, journal publishers and funding agencies to require quality standards to improve translatability of preclinical findings.
\end{abstract}

Keywords: Animal models, mice, mdx, preclinical drug evaluation, guidelines

\section{INTRODUCTION}

In the last few years, several publications, editorials and commentaries have addressed the issue of transparency and reproducibility in research. Robustness of results is particularly important in research on animal models with the aim of assessing the potential efficacy of a treatment for patients, as such data may serve as the basis for the hope of patients and for large and expensive clinical trials. The low predictive power of preclinical studies to translate into successful clinical trials has caused frustration in the patients' as well as in the researchers' communities [1-5]. The poor translation of preclinical research is even more criti-

*Correspondence to: Raffaella Willmann, Swiss Foundation for Research on Muscle Diseases, Cortaillod, Switzerland. E-mail: raffaella.willmann@unibas.ch. cal in neuromuscular diseases because these disorders are rare and have a limited number of patients: unsuccessful trials will prevent patients from participating in other trials and valuable resources and energy are drained in these costly and complex multi-site clinical trials. The rather poor predictability is often used to incriminate animal models of disease in general as poor predictors of drug efficacy. Surely, the response of animal models to drugs and treatments does not precisely reflect the response in patients; however, animal models are and have been in the past very valuable tools for understanding the complex and dynamic nature of disease progression, and for developing therapeutic concepts. Thus, testing potential treatment options in animal models is certainly the most appropriate way to select drug candidates and establish their potential clinical benefit in humans. However, the lack of rigorous and consistent design of the preclinical tests conducted 
with animal models can be one reason that contributes to the failure in translation [6]. This review will discuss a few, simple measures to minimize experimental variations and explore possibilities and obstacles linked to this effort.

\section{EXPLORATORY AND CONFIRMATORY STUDIES}

In the process of identifying new therapeutic targets and developing potential treatments, many experimentations aim at understanding a given physiological pathway, generate hypotheses, investigate new possibilities or methods. At a later time point, a confirmatory study is necessary to provide compelling evidence that the treatment is worth being tested in humans and that it justifies the enormous financial and emotional effort bound to a clinical trial [7]. Confirmatory preclinical animal studies obviously need a high quality standard and a careful study design, while these aspects are less important in exploratory studies aimed at generating data for further development. Many of the published preclinical studies are, however, conducted as exploratory studies [8] but are then used to promote and sustain the conduct of a clinical trial. In conclusion, while freedom and flexibility should be granted in exploratory studies, the implementation of rigor and quality in confirmatory preclinical animal studies would be of great benefit for the planning of successful clinical trials especially for rare neuromuscular diseases.

This however requires a wider awareness of the scientific community and of journals' editors in order to optimize the efforts in this delicate translational attempt.

\section{QUALITY IN CONFIRMATORY PRECLINICAL STUDIES FOR NEUROMUSCULAR DISEASES}

The problematic of bench research delivering efficacy data that turn out not to show any efficacy in patient studies is not new and was discussed thoroughly in the communities of both rare and common diseases $[1,5,6,9-11]$. A general problem that makes it difficult to compare these studies and draw conclusions is the incomplete reporting of experimental settings or data generation. There is a common consensus on the assumption that transparency, reproducibility and finally also the predictive power of results can be significantly increased by adopting standards in experi- mental design like randomization and blinding, sample size estimation and data handling $[6,12,13]$.

In a detailed survey of preclinical efficacy/proofof-concept studies on the mdx mouse model, the discrepancy between this common consensus and reality becomes evident. The study, initiated and financed by Parent Project Muscular Dystrophy USA, analysed almost 200 papers published between 2000 and 2011 and recorded data reporting and drug study results. Of all papers, randomization of mice in control and treated groups was described in only $13 \%$ of the cases, and parameters were assesses in a blinded way in only $7.4 \%$ of the papers (V. Malik and R. Willmann, personal communication). Only 2 papers reported sample size calculations to justify the number of mice chosen; in others, sample size was less than 8 mice per group $(40 \%)$ or was even not reported $(14 \%)$. More than $40 \%$ of the papers did not report mouse sex and $12.7 \%$ reported the use of mixed sexes, suggesting that there is no consensus on detailed reporting of preclinical data in the published papers. Finally, only about $16 \%$ reported the food regimen and brand used, despite the fact that food composition can considerably vary between suppliers and influences metabolism and efficacy of pharmaceutical compounds. For instance, soy protein has an impact on cellular responses and gene expression [14], and 5 of the 6 diets compared in fact used soy beans as protein source (R. Willmann, personal observation).

In addition to transparency in reporting, animalspecific best practices that take account of bias related to a specific animal model should be considered: this issue was addressed for mouse models of Duchenne Muscular Dystrophy [15] [5, 16] and also for other, non neuromuscular diseases $[10,11]$. Comparing recommendations suggested by the different authors $[15,17-19]$ and guidelines adopted by single institutions (see for instance http://www.nih.gov/about/ reporting-preclinical-research.htm, based on [6]), it seems that some general rules of transparency should apply to all confirmatory preclinical studies, independently of the disease addressed. These are summarized in Table 1 and help structuring the confirmatory preclinical study similar to guidelines used in patient trials.

One important aspect that differs between the diseases studied is the choice of the animal model and of the efficacy readouts that reflect human outcome measures for that condition. In the case of neuromuscular diseases, histological and biochemical data serve to quantify changes at the molecular level and can be determined with more precision and with larger sample sizes. Functional assays, including the assessment 
Table 1

Suggested steps to consider in the planning and reporting of a confirmatory preclinical study

\begin{tabular}{ll}
\hline Study phase & Actions \\
\hline Study design & Identify appropriate outcome measures \\
& Determine appropriate sample size required \\
& Determine inclusion and exclusion criteria \\
& Determine appropriate species, sex, age and duration of intervention \\
Animal handling & Include placebo and wild type controls \\
& Determine litter size \\
& Randomize animals in groups \\
Results collection & Plan blinded experiment conduct and evaluation of results \\
& Perform experiments at the same time of the day preferably by the same person \\
& Use standard protocols to quantify outcome measures \\
& Replicate experiments \\
Interpretation & Determine procedures for dealing with dropouts or deaths \\
& Determine significance \\
& Determine dose-response results \\
& Use appropriate statistical analysis \\
& Report genetic background, age, sex, number of animals \\
& Report negative results \\
Reporting & Report husbandry conditions and diet \\
& Report raw data
\end{tabular}

of locomotion, muscle force or overall health, deliver information that may be of importance in the evaluation of the benefit in clinical trials and might be beyond any real improvement of the disease condition. Therefore, a balanced choice of in-vitro and in-vivo assays is recommended in mouse models for neuromuscular diseases. Beyond the choice of outcome measures, the need of standardized protocols to assess them became evident already some years ago. For some animal models, such protocols are available [20, 21]. The use of standard operating procedures (SOPs) offers the possibility of evaluating variability and calculating the required sample size by power analysis for a specific assay, and to determine the natural history and reference data for a parameter in a given model.

In the example of the mdx mouse model for DMD, the experimental use of the animal model underwent a progressive increase over the years. A search in PubMed simply using "mdx mouse", reported a total of 2409 articles from 1984 (the date this model was first described) up to October 2014; the number of publications steadily increased from 2000 to 2007 and then stabilized at more than 100/year. In parallel, the focus on standardization of experimental approaches started in 2007 and was a dynamic step-wise process which required some time to reach a consensus $[5,16]$. Under the assumption that less than half of the published articles deals with "therapeutics", the full acceptance of using SOPs for pre-clinical drug tests in such a large community may require time and a clear communication on how improving standardization may help translational activity.
Another issue that has to be considered, which is not directly related to the animal model, is the fact that most of the studies conducted face the need of publication. Journal editors mainly require two main things: a) novelty and b) impressive results. A novel study, even when based on a strong rationale and an important hypothesis will hardly be published when negative results are obtained. Impressive confirmatory results that lack novelty may encounter similar problems. Related to this aspect is the need (often required by reviewers and editors) of having suggestive titles even for proof-of-concept studies, with the idea that this may attract more readers and citations compared to a more neutral one. It is easy to conclude about the immediate consequences of these aspects, especially for orphan diseases in which the unmet clinical need and the expectation of a therapy are compelling. A journal policy oriented toward the publication of well conducted and well described studies, independently of their negative or positive results, is already implemented by journals like Plos One. The adoption of this model by editors in the neuromuscular field would undoubtedly help in progressing research and in avoiding unnecessary efforts and investments.

\section{THE ROLE OF THE RESEARCH COMMUNITY AND STAKEHOLDERS IN IMPROVING TRANSLATION}

The responsibility to improve the predictive power of preclinical experiments should not solely depend 
on the decision of single laboratories. It is a combined effort of research community, funding organization and journal editors that will lead to the desired change. In 2011, a meeting in Washington D.C. to discuss such responsibilities resulted in guidelines that will be used by NIH in evaluating grants ([22], see also http://grants.nih.gov/grants/guide/ notice-files/ NOTNS-11-023.html). The TREAT-NMD Advisory Committee for Therapeutics (TACT), set up by the EU-funded Network TREAT-NMD as a tool to help promising therapies to reach patients as quickly as possible, provides guidance on advancing new therapies for neuromuscular diseases and requires the use of SOPs where applicable. Many companies involved in the development of therapeutics for DMD are aware of the need of proper standardization of preclinical tests, and require SOP adherence when collaborating with academia or CROs. Nature and Science Translational Research published a checklist for authors that includes reporting and statistical guidelines that need to be followed in the submitted manuscripts [23, 24]. Recently, a workshop was organized by TREAT-NMD to provide a coordinated response to the public consultation on the draft guideline "Clinical investigation of medicinal products for the treatment of DMD and BMD" released by the European Medicine Agency (EMA) in 2013. The workshop brought together academics, patient representatives, industry representatives and individual experts. Among the various points, the draft guidelines of EMA pointed out that the proposed mechanism of action of any new product should be described and discussed in relation to possible testing in available animal models, which were, however, described as poor. One issue of the workshop focused therefore on animal models and it was emphasized how the effort of TREAT-NMD in implementing and disseminating proper use of valuable animals models by means of SOPs served to initiate the discussion on quality and how transparency, blinding and power calculation, along with a better distinction between primary and secondary outcome measures, may improve predictability of results [25].

As shown by these few examples, awareness on the issue of study quality can be raised if journals, grant funding organisations and regulatory agencies insist on standards for preclinical studies. Pre-clinical research may learn from what has been previously discussed for clinical trials. For instance, the publication of the consolidated standards for reporting trials (CONSORT statement) in 2001 resulted in a strong improvement as to how trials were reported in journals [26]. This is also reflected by the position taken by the International Committee of Medical Journal Editors, that paved the way toward having transparent public reports of clinical trials and their outcome [27, 28]. In 2004, an editorial of this Committee underlined the risk of bias when a selective publication policy is used. It was emphasized that researchers and journal editors are generally keen to publish trials that show either a large effect of a new treatment (positive trials) or noninferiority results (equivalence of two approaches to treatments) and that they are generally less interested in publishing negative or inconclusive trials. The editorial pushed for a transparent reporting of clinical trials, with exact mentioning of existing evidences in the specific field at the time of article submission to the associated medical journals, in order to reduce the potential bias from selective publication; and underlined that, rather than a single trial, it is usually a body of evidence from many studies that changes medical practice. Such an effort is needed also for pre-clinical tests, especially when the limited patient population requires a careful selection of best candidates for human testing.

The issue of investing time and funding in defining best practices and standard protocols for animal models of diseases definitely deserves more attention. Specific grants by funding agencies and international projects should be dedicated to the characterization of mouse and other animal models (see also [29]), their natural histories and variability of analysis; and to workshops aimed at finding consensus on animal-specific guidelines and protocols.

\section{CONCLUSION}

The development of therapeutic approaches for rare neuromuscular diseases is an urgent medical need coupled with expectations and hopes of an overall significant group of patients. Therefore, the applicability of preclinical findings to human trials is of particular importance. Exploratory and proof-of-concept studies where drugs are used to validate a pathology-related pathway, are often translated too quickly into clinical trials if results showed some evidence of beneficial modulation of the diseases in the mouse. This makes it difficult to prioritize candidates for clinical trials on a limited patient population and increases the risk of clinical failure for testing a drug which in fact has not robust pre-clinical evidence. In addition, a drug failing a clinical test often leads to the conclusion that the animal model is of limited usefulness because not representative of the human disease. Rather, the in-depth 
knowledge of the model will help identifying potential targets and predicting if a drug may work in humans, and preclinical studies conducted with the appropriate rigor will help understanding which signs or symptoms are more likely to be improved in humans. The use of SOPs for functional and biochemical assays and of common guidelines in the conduct of preclinical confirmatory studies is therefore the way to pursue and to improve to obtain more comparable results between different laboratories and a higher translational success.

The aim of this review was to try to reconcile different positions, and to fill the gap between scientific community, clinical needs and editorial policy. This may help to better harmonize the scientific progress reached by basic science with the investments into drug development and clinical studies and to possibly improve the final common goal: a better therapy for neuromuscular disorders.

\section{REFERENCES}

[1] van der Worp, H. B., et al. Can animal models of disease reliably inform human studies? PLoS Med. 2010; 7(3): e1000245.

[2] Vesterinen, H. M., et al. Improving the translational hit of experimental treatments in multiple sclerosis. Mult Scler. 2010; 16(9): 1044-1055.

[3] Prinz, F., Schlange, T., Asadullah, K. Believe it or not: How much can we rely on published data on potential drug targets? Nat Rev Drug Discov. 2011; 10(9): 712.

[4] Benatar, M. Lost in translation: Treatment trials in the SOD1 mouse and in human ALS. Neurobiol Dis. 2007; 26(1): 1-13.

[5] Willmann, R., et al. Enhancing translation: Guidelines for standard pre-clinical experiments in mdx mice. Neuromuscul Disord. 2012; 22(1): 43-49.

[6] Landis, S. C., et al. A call for transparent reporting to optimize the predictive value of preclinical research. Nature. 2012; 490(7419): 187-191.

[7] Kimmelman, J., Mogil, J. S., Dirnagl, U. Distinguishing between exploratory and confirmatory preclinical research will improve translation. PLoS Biol. 2014; 12(5): e1001863.

[8] Mogil, J. S., Simmonds, K., Simmonds, M. J. Pain research from 1975 to 2007: A categorical and bibliometric meta-trend analysis of every Research Paper published in the journal, Pain. Pain. 2009; 142(1-2): 48-58.

[9] Begley, C. G., Ellis, L. M. Drug development: Raise standards for preclinical cancer research. Nature. 2012; 483(7391): 531-3.

[10] Shineman, D. W., et al. Accelerating drug discovery for Alzheimer's disease: Best practices for preclinical animal studies. Alzheimers Res Ther. 2011; 3(5): 28.
[11] Ludolph, A. C., et al. Guidelines for preclinical animal research in ALS/MND: A consensus meeting. Amyotroph Lateral Scler. 2010; 11(1-2): 38-45.

[12] Kilkenny, C., et al. Improving bioscience research reporting: The ARRIVE guidelines for reporting animal research. PLoS Biol. 2010; 8(6): e1000412.

[13] Festing, M. F., Altman, D. G. Guidelines for the design and statistical analysis of experiments using laboratory animals. ILAR J. 2002; 43(4): 244-258.

[14] Stauffer, B. L., et al. Soy diet worsens heart disease in mice. J Clin Invest. 2006; 116(1): 209-216.

[15] De Luca, A. Pre-clinical drug tests in the mdx mouse as a model of dystrophinopathies: An overview. Acta Myol. 2012; 31(1): 40-47.

[16] Grounds, M. D., et al. Towards developing standard operating procedures for pre-clinical testing in the mdx mouse model of Duchenne muscular dystrophy. Neurobiol Dis. 2008; 31(1): 1-19.

[17] Grounds, M. D. Two-tiered hypotheses for Duchenne muscular dystrophy. Cell Mol Life Sci. 2008; 65(11): 1621-1625.

[18] Spurney, C. F., et al. Preclinical drug trials in the mdx mouse: Assessment of reliable and sensitive outcome measures. Muscle Nerve. 2009; 39(5): 591-602.

[19] Kornegay, J. N., et al. Pharmacologic management of Duchenne muscular dystrophy: Target identification and preclinical trials. ILAR J. 2014; 55(1): 119-149.

[20] Nagaraju, K., Willmann, R. Developing standard procedures for murine and canine efficacy studies of DMD therapeutics: Report of two expert workshops on "Pre-clinical testing for Duchenne dystrophy": Washington DC, October 27th-28th 2007 and Zurich, June 30th-July 1st 2008. Neuromuscul Disord. 2009; 19(7): 502-6.

[21] Willmann, R., Dubach, J., Chen, K. Developing standard procedures for pre-clinical efficacy studies in mouse models of spinal muscular atrophy: Report of the expert workshop "Pre-clinical testing for SMA", Zurich, March 29-30th 2010. Neuromuscul Disord. 2010; 21(1): 74-7.

[22] Couzin-Frankel, J. When mice mislead. Science. 2013; 342(6161): 922-923, 925.

[23] Journals unite for reproducibility. Nature. 2014; 515(7525): 7.

[24] McNutt, M. Journals unite for reproducibility. Science. 2014; 346(6210): 679 .

[25] Lynn, S., et al. Measuring clinical effectiveness of medicinal products for the treatment of Duchenne muscular dystrophy. Neuromuscul Disord. 2014.

[26] Kane, R. L., Wang, J., Garrard, J. Reporting in randomized clinical trials improved after adoption of the CONSORT statement. J Clin Epidemiol. 2007; 60(3): 241-249.

[27] De Angelis, C., et al. Clinical trial registration: A statement from the International Committee of Medical Journal Editors. Lancet. 2004; 364(9438): 911-912.

[28] Mosconi, P., Roberto, A. Open-access clinical trial registries: The Italian scenario. Trials. 2012; 13: 194.

[29] Perrin, S. Preclinical research: Make mouse studies work. Nature. 2014; 507(7493): 423-425. 